\title{
How plate positioning impacts the biomechanics of the open wedge tibial osteotomy; A finite element analysis
}

\author{
L. D. BLECHA $\dagger$, P. Y. ZAMBELLI†, N. A. RAMANIRAKA †, P.-E. BOURBANף, J.-A. MÅNSONף and D. P. PIOLETTI†*
}

$\dagger$ EPFL, Laboratory for Orthopedic Research, Lausanne, Switzerland

\$Hôpital Orthopédique de la Suisse Romande, Lausanne, Switzerland

१EPFL, Laboratory of Polymer and Composite Technology, Lausanne, Switzerland

(Received 5 March 2005; in final form 24 August 2005)

\begin{abstract}
A numerical model of the medial open wedge tibial osteotomy based on the finite element method was developed. Two plate positions were tested numerically. In a configuration, (a), the plate was fixed in a medial position and (b) in an anteromedial position. The simulation took into account soft tissues preload, muscular tonus and maximal gait load.

The maximal stresses observed in the four structural elements (bone, plate, wedge, screws) of an osteotomy with plate in medial position were substantially higher (1.13-2.8 times more) than those observed in osteotomy with an anteromedial plate configuration. An important increase (1.71 times more) of the relative micromotions between the wedge and the bone was also observed. In order to avoid formation of fibrous tissue at the bone wedge interface, the osteotomy should be loaded under $18.8 \%(\sim 50 \mathrm{~kg})$ of the normal gait load until the osteotomy interfaces union is achieved.
\end{abstract}

Keywords: Osteotomy; Biomechanics; Finite element analysis; Bone tissue engineering; Plate position

\section{Introduction}

Medial open wedge tibial osteotomy is a current surgical intervention for the correction of varus knee deformities. It is preferred to the lateral closing wedge osteotomy as numerous complications shall be avoided such as neurological complication (Hernigou and Ma 2001), compartment syndrome (Staubli et al. 2003), lateral muscle detachment, proximal fibula osteotomy and leg shortening (Lobenhoffer et al. 2004). In several clinical studies (Badhe and Forster 2002, Koshino et al. 2003) better score on open wedge than on closing wedge osteotomy were observed. Moreover, the open wedge osteotomy is preferred as the difficulties encountered when performing a subsequent total knee arthroplasty are reduced (Hernigou and Ma 2001).

Earlier no internal fixation or plaster was used for maintaining the wedge in place. It was thought that the wedge would be held by compressive forces due to elongation of the surrounding soft tissues. But displacement of the wedge with loss of correction was observed (Hernigou et al. 1987). To overcome this complication, an internal fixation composed of a plate and screws were used for all osteotomies. In recent open wedge varus osteotomy studies (Hernigou and Ma 2001, Badhe and Forster 2002, Koshino et al. 2003, Staubli et al. 2003), the use of internal or external fixation at least during 4 months postoperatively were generalized. Although, some clinical studies proposed a specific positioning of the fixation and wedge (Hernigou and Ma 2001, Koshino et al. 2003, Staubli et al. 2003), no comparison between different layouts was found neither in these studies, nor in finite element investigations (Sato and Morishita 1999, Schmitt et al. 2001). From a biomechanical viewpoint, the spatial distribution of the structural elements composing the osteotomy (wedge, plate, bone) plays a key role. Indeed these elements are responsible for the structural stability of the osteotomy. Their relative positions with regard to the applied physiological loads have an important impact on the stress distribution and micromotion magnitude at the bone-wedge interface. Although, no clinical studies reported wedge failure nor failure due to fibrous bone formation at the bone wedge interface, the load path shall be optimized in order to minimize stresses and micromotions at the bone-wedge interface. Indeed, large micromotions seem to take place at this interface

*Corresponding author. E-mail: dominique.pioletti@epfl.ch

Computer Methods in Biomechanics and Biomedical Engineering ISSN 1025-5842 print/ISSN 1476-8259 online (C) 2005 Taylor \& Francis http://www.tandf.co.uk/journals DOI: $10.1080 / 10255840500322433$ 
as Hernigou et al. (Hernigou and Ma 2001) observed lucent line at the bone-wedge interface on radiographs, indicating presence of fibrous tissue at this interface.

The goal of this study was to evaluate how would be modified the stress distribution and micromotion magnitude when fixing the supporting plate (SP) in an anteromedial position rather than in a medial position. The two configurations were tested numerically with help of a finite element model of the medial open wedge tibial osteotomy. The simulation took into account soft tissues preload, muscle tonus and maximal gait load. The wedge was assumed to be made of acrylic surgical cement and was placed in posteromedial position which corresponds to the current clinical practice (Hernigou and Ma 2001).

\section{Methods}

\subsection{Finite element mesh}

Using the commercial software ABAQUS/Standard 6.2-1 (HKS Inc., Newpark, USA), a mathematical model based on the finite element method (FEM) was built to investigate numerically the open-wedge osteotomy. The external contour of an intact tibia was reconstructed from CT slices of a patient of 35 years old without apparent pathology. It is thus assumed that the tibia of a patient with various deformation has a similar spatial bone density distribution than a healthy knee. The optimal size of the pixel was $0.39 \mathrm{~mm}$, with a resolution of $512 \times 512$ pixels and CT transversal slices are obtained every $0.7 \mathrm{~mm}$. The 3D geometry was generated by AMIRA software (Konrad-Zuse-Zentrum für Informatik Berlin, Germany) and then transferred to PATRAN software (MacNeal-Schwendler, South Coast Metro, California, USA) for FE mesh generation. It was estimated that such a procedure allows a geometrical reconstruction within an error of 2 pixels, i.e. $0.78 \mathrm{~mm}$ (Büchler et al. 2002). Using the automatic mesh generator of PATRAN, first order hexahedral volume elements (C3D8) were created. Thorough attention was paid to have at least two volume elements through the cortical bone depth, in order to get a good numerical representation of the density step between cancellous and cortical bone (Rubin et al. 1993). The osteotomy was then performed virtually under the supervision of a senior orthopedist, and a correction angle of $10^{\circ}$ was applied. A wedge with a cross-section corresponding to one forth of the osteotomy section was laid in the posteromedial position (figure 1), which correspond to the current clinical practice (Hernigou and Ma 2001). The interfaces between the wedge and the tibia were modeled by contact surfaces which allow separation and friction. The tangential friction law was based on Coulomb's criterion for delimiting adherence from friction and a non-associated slip rule governing the slip velocity. The SP was reconstructed geometrically from a standard stainless steel T-plate (Synthes), and modeled by quadrilateral shell elements (S4R5). It was screwed to the tibial head by four titanium M4 $\times 55$ screws, which were modeled by beam elements (B31). In order to model the load transfer at the interface between the screw and the bone, multipoint elements (LINEA) were used to connect each node of the screw (beam elements) to the neighbouring nodes of the bone located within a disc of $4 \mathrm{~mm}$ diameter. Thus, the bending stiffness of the screws was modelled accurately with the only assumption that the radial stiffness of the screws was assumed infinite. The connection of the screw head to the T-plate was achieved similarly. The leg of the T-plate was fixed to the main tibia part by two screws which were modeled identically as the screws fixing the T-plate to the tibia head. Two SP positions were studied; (a) the plate was positioned on the lateromedial axis, in medial position; (b) the plate was positioned at half distance between the posteroanterior and lateromedial axis, in anteromedial position (figure 1).

The whole osteotomy model was composed of 98 beam elements (B31), 676 shell elements (S4R5) and 25496 volume elements (C3D8).

\subsection{Material properties}

The constitutive laws for both cortical and cancellous bones were assumed to be linear elastic, inhomogeneous and with transverse isotropic symmetry. This was found

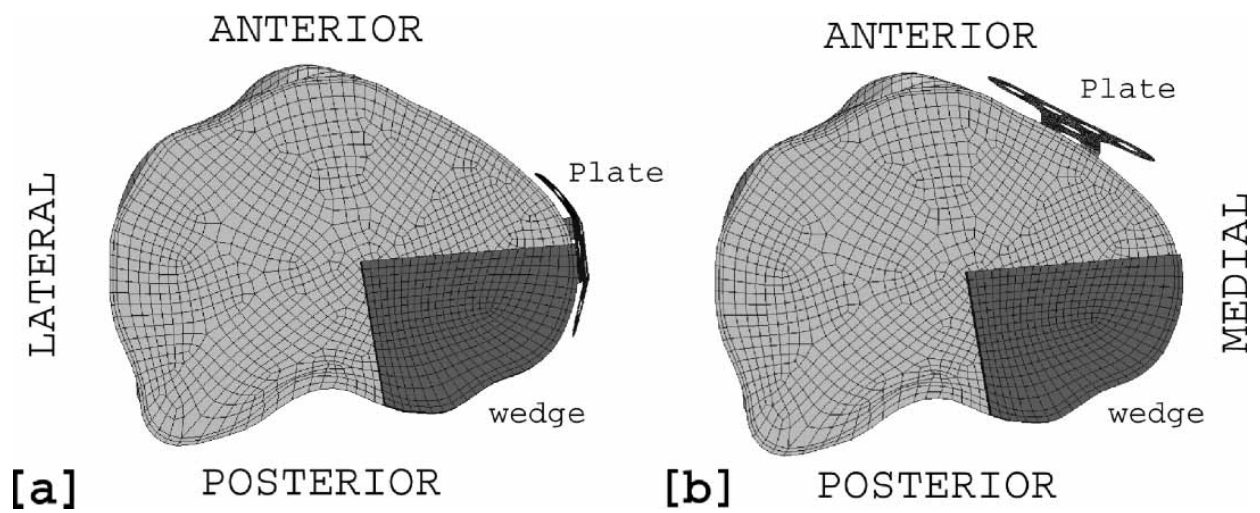

Figure 1. Plate positioning - Top view of the tibial osteotomy without tibia head with a SP in (a) medial position, and in (b) anteromedial position. 
to be a reasonable assumption (Williams and Lewis 1982, Rakotomanana et al. 1992, Rubin et al. 1993). Inhomogeneity of bone was taken into account by considering a quadratic relation between elastic moduli, shear modulus and apparent density (Rice et al. 1988, Linde et al. 1991). The elastic constants were taken form the literature (Reilly and Burstein 1975, Cowin 2001).

The friction coefficient between the wedge and the tibia was assumed to be similar to the one observed at a bonebone contact. Van Frauenhofer et al. (Shockey et al. 1985, Von Fraunhofer and Schaper 1985) have quantified the translational and the rotational friction coefficient between two bony structures between 0.5 and 0.7 .

The wedge and T-plate materials were considered linear elastic, homogeneous and isotropic. The T-plate was made of surgical stainless steel (AISI 316L) which had an elastic modulus of $200 \mathrm{GPa}$ and Poisson ratio of 0.3 . The elastic constants of the wedge were assumed to be similar to those of commercial acrylic surgical cement, often used in open wedge osteotomy. Harper et al. (Harper and Bonfield 2000) reported an average Young's modulus of $3 \mathrm{GPa}$. A Poisson ratio near incompressibility were assumed, i.e. 0.45.

\subsection{Loading and boundary conditions}

The loading occurred in two steps corresponding to (a) the surgical intervention induced-loads and (b) physiological loads taking place after surgery (figure 2). Firstly, the tibia was opened with a correction angle of $10^{\circ}$, corresponding to the averaged correction currently performed. The wedge was placed in-between the tibia parts and held in position by the compression loads due to the elongation of the remaining intact cortex, medial collateral and patellar ligaments. A dedicated FE model was created to evaluate the periosteum elongation loads and is described in the following section. In addition to the surgical intervention induced loads, femorotibial contact loads due to muscular tonus were further compressing the wedge. The tonus was estimated to be $400 \mathrm{~N}$ (Taylor et al. 1997).
Secondly, once the T-plate was screwed, the osteotomized tibia was loaded by postoperative physiological loads. The gait loads were chosen because it is the most constraining activity allowed post-operatively. These are well known in terms of total resultant joint load (Taylor et al. 1997, Hurwitz et al. 1998) and a maximal gait load of 3.5 times the body weight (BW) was assumed. It takes place at small knee flexion angle, therefore, full extension was assumed. The femorotibial contact load distribution was obtained from a FE model (figure 2) developed by Ramaniraka et al. (Ramaniraka et al. 2003). As the aim of the open wedge tibial osteotomy is to restore a favourable load bearing axis of the lower limb, it was assumed that a physiological load repartition between the lateral and medial plateau of $40-60 \%$ was achieved. The locations of the maximal contact stress on the tibial plateau were similar to those reported in the literature (Hill et al. 2000, Wretenberg et al. 2002, Donahue et al. 2003). Applying tibiofemoral loads of a healthy knee on an osteotomized tibia neglected the effect of tibial head deformation on the tibiofemoral contact load distribution. Transposition of the femorotibial contact forces from the complete knee model to the osteotomy model was carried out relative to their intrinsic coordinate system for the tibial plateau (Pioletti et al. 1998a) and normalized to their cross section inertia.

\subsection{Periosteum elongation model}

This model was meant to obtain numerical values of the jaw forces induced by the opening sequence of the osteotomy. They were mainly due to the elongation of the remaining intact cortex, medial collateral and patellar ligaments which exerted a stabilizing compression force on the wedge (Hernigou et al. 1987). It was assumed that the elongation of an intact periosteum of constant thickness was representative of the reality. Furthermore, the periosteum was taken as perfectly dissociated from the tibia (no friction) on a given height and on the entire

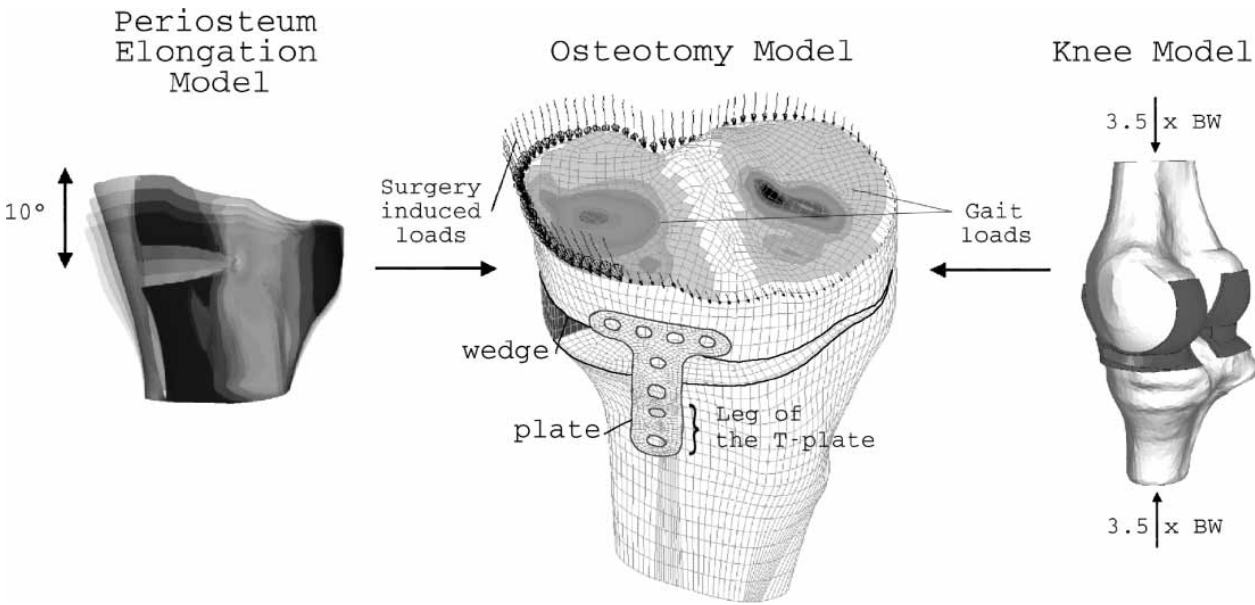

Figure 2. Medial open wedge tibial osteotomy finite element model: The loads due the elongation of the periosteum after the opening the tibia were evaluated with a simplified periosteum elongation model (left) and applied to the osteotomy model (middle). The maximal gait loads were calculated with an entire knee model (right) and than applied to the osteotomy model (middle). 
circumference. The height corresponded to the length of the vertical incision carried out by the surgeon, along which the cortex was detached from the bone with a chisel. A second incision was performed horizontally on the anteromedial side of the tibia. This opening enables the surgeon to cut the tibia and to insert the wedge inbetween the tibia parts.

The external contour of the tibia was defined by the reconstructed geometry from CT slices. On the height of the incision, deformable quadrilateral shell elements (S4R5) of a $0.5 \mathrm{~mm}$ constant thickness were used to mesh the periosteum. The local stiffness variations of the peiosteum due to ligament attachment were not taken into account leading to locally underestimated jaw force. The nodes of the elements at the incision vicinity were not equivalenced, forbidding load transfer. The entire model was composed of 2036 shell elements (S4R5).

The periosteum was considered homogeneous linear elastic. As the mechanical properties of soft tissue vary significantly between individuals (Pioletti et al. 1998b, Han et al. 2003), averaged values found in the literature were used (Uchiyama et al. 1998, Popowics et al. 2002). The tensile modulus was set to $80 \mathrm{MPa}$ and Poisson ratio to 0.495 .

In order to model the opening of the osteotomy, the element nodes at the upper limit of the periosteum were forced to move of values corresponding to a rotation of $10^{\circ}$ around the bone hinge formed by the remaining cortical bone at osteotomy level. The nodes at the lower limit were fixed in translation.

A total force of $821 \mathrm{~N}$ was exerted by the elongated cortex. This load was not continuously distributed along the tibia circumference. Stronger closing loads were observed on the posterior side than on the anterior side of the tibia. The asymmetry and the order of magnitude of the jaw loads were confirmed by surgeons who reported subjectively to apply a rather high opening force. Assuming a maximal hand grip force of $80 \mathrm{~kg}$ together with a level of arm of 2 w.r.t. the cortex closing force, a maximal opening force of $1600 \mathrm{~N}$ may be exerted. A subjectively determined rather high force may reasonably be related to $50 \%$ of the maximal hand grip force of the surgeon. The calculated forces due to periosteum elongation were then applied on the osteotomy global model (figure 2).

\section{Results}

\subsection{Micromotions and displacement}

The maximal micromotion at the interface between the bone and the wedge was greater in osteotomy with SP in medial position than osteotomy with anteromedial plate position (figure 3). At $3.5 \mathrm{BW}$, a factor 1.71 between the two configurations was observed. The maximal micromotion magnitude for the medial plate position was 1230 and $720 \mu \mathrm{m}$ for the anteromedial plate configuration. The maximal micromotion for both configurations was

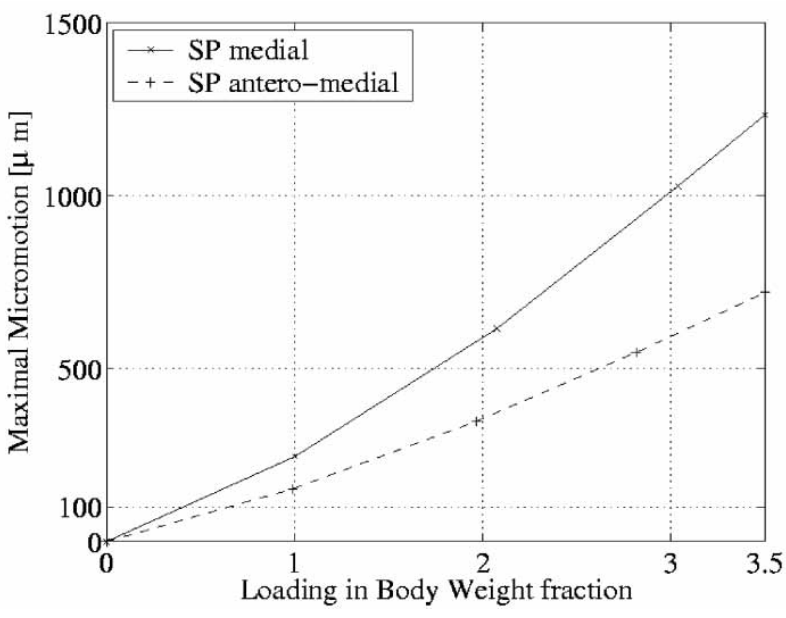

Figure 3. Maximal micromotion at the interface between bone and wedge in function of the loading in body weight fraction for two different SP positions.

located at the lower interface between the wedge and the bone.

The osteotomized tibia with SP in medial position showed the maximal displacement. It was located on the tibial head and its magnitude was $8.3 \mathrm{~mm}$ at $3.5 \mathrm{BW}$. This value decreased by a factor $1.38-6.0 \mathrm{~mm}$ for an anteromedial plate position. The movement associated with the displacement can be decomposed in a rotation around the remaining bone at osteotomy level and a rotation around the lateromedial axis. This last movement was more pronounced in a medial plate configuration.

\subsection{Stress distribution}

The maximal von Mises stresses observed in the osteotomized tibia was the greatest when the SP was fixed in medial position (figure 4). Higher maximal stresses were also observed in the wedge, the SP and the screws for tibial osteotomy with medial SP. At 3.5 BW,

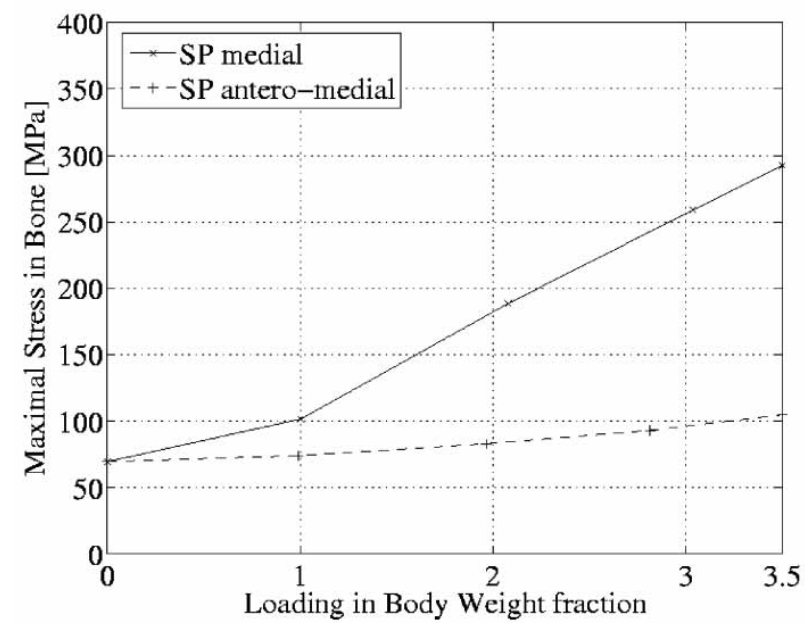

Figure 4. Maximal von Mises stress observed in the tibia bony structure in function of the loading in body weight fraction for two different SP positions. 
the maximal stress in the bone was 2.78 times higher in a medial plate configuration than an anteromedial configuration, with magnitudes of 292.3 and $104.8 \mathrm{MPa}$, respectively. In the wedge, a factor of 1.45 was found between osteotomy with the plate in medial position $\left(\sigma_{\max }=168 \mathrm{MPa}\right)$ and the osteotomy with the plate in anteromedial position $\left(\sigma_{\max }=116 \mathrm{MPa}\right)$. Finally, a decrease of the maximal stress in the SP of a factor 1.40 and in the screws of a factor 1.13 was also observed between a medial plate position $\left(\mathrm{SP} \sigma_{\max }=2072 \mathrm{MPa}\right.$; screws $\left.\sigma_{\max }=122 \mathrm{MPa}\right)$ and an anteromedial plate position $\left(\mathrm{SP} \sigma_{\max }=1485 \mathrm{MPa}\right.$; screws $\left.\sigma_{\max }=108 \mathrm{MPa}\right)$.

The location of the maximal stress on bone for both configurations was found to be under the lower medial corner of the wedge. The maximal stress on the wedge was concentrated in the medial corner of the wedge. The most constrained region of the fixation system composed of the T-plate and the screws was located at the junction between upper screw fixing the leg of the T-plate to the main tibia part.

\subsection{Effect of friction coefficient}

The variation of the friction coefficient at the interface between the wedge and bone had small impact on the stress distribution. Increasing the friction coefficient from a value of 0.5 to a value of 0.7 caused a maximal stress variation in the SP of a factor 1.07. The stress variations observed in the bone and wedge were even smaller. Similar increase of the friction coefficient resulted in a decrease by a factor 1.19 of the maximal micromotion (figure 5).

\section{Discussion}

The goal of this study was to evaluate the variation of stress level and micromotion magnitude due to different positions of the SP in an open wedge osteotomy. It was

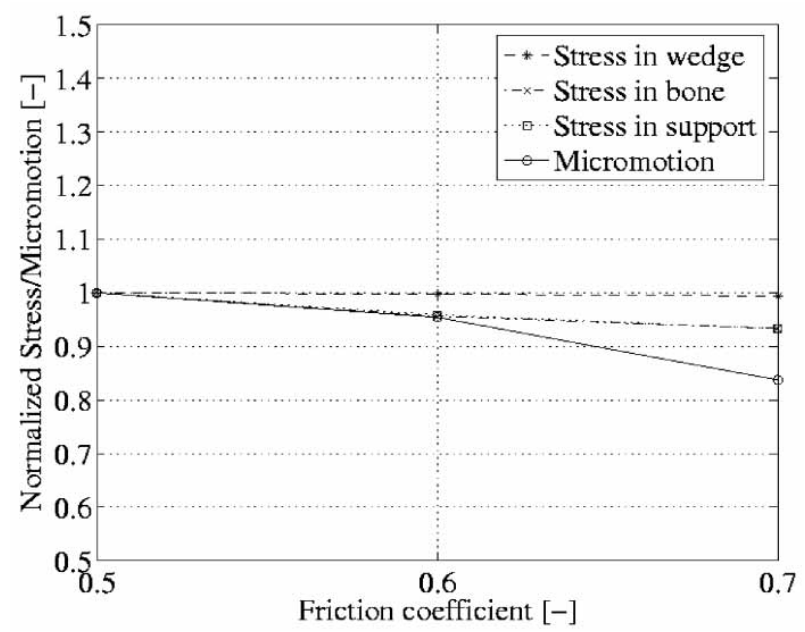

Figure 5. Impact of the interface friction coefficient on the maximal stresses and maximal micromotions. found that when the plate was in a medial position, the maximal stresses observed in the four structural elements (bone, plate, wedge, screws) were substantially higher than by an anteromedial plate configuration. Factors between 1.13 and 2.8 depending on the elements were observed between the two configurations at full weight bearing. Placing the SP in an anteromedial position was limiting the maximal stress. At $3.5 \mathrm{BW}$, the maximal stress in the bone and in the SP was under the ultimate stress generally reported for the bone $(104<190 \mathrm{MPa})$, and surgical stainless steel plate $(1485<1500 \mathrm{MPa})$. Instead, the ultimate stress for bone was exceeded after $2 \mathrm{BW}$ in the case of a medial plate position. The very high stresses calculated in the wedge, plate and bone took place at maximal gait load and are local. Hopefully, these loads are most of the time avoided in clinic by prescribing a partial loading of the knee (up to $20 \mathrm{~kg}$ ) until a clear evidence of the osteotomy consolidation was seen on radiographs.

A great decrease (1.71 times less) of the micromotions between the wedge and the bone were calculated when the plate was moved from a medial to an anteromedial position. However, the level of micromotion at $3.5 \mathrm{BW}$ was well above the limit of $100 \mu \mathrm{m}$ after which took place the formation of fibrous tissue at the bone/implant interface described in the literature (Pilliar et al. 1986, Soballe et al. 1992a, Soballe et al. 1992b, Soballe 1993, Jasty et al. 1997, Büchler et al. 2002). Maximal micromotions less than $100 \mu \mathrm{m}$ was calculated (figure 3) for open wedge osteotomy with a plate in anteromedial position and loaded under $0.65 \mathrm{BW}$, which corresponds to a partial loading of the knee of $18.8 \%(\sim 50 \mathrm{~kg})$.

It was shown that the friction coefficient had small impact on the stress and micromotion values. A weak decreasing behavior of the maximal stress and micromotion with increasing friction coefficient was observed (figure 5). Thus, the most constraining case was identified to be an open wedge osteotomy calculated with friction coefficient of 0.5 .

During gait cycle, the impact of the plate position on the biomechanics of the osteotomy was maximal when constrained by the maximal gait load. As it took place at small flexion angle, the femorotibial contact forces were the most anterior. During the rest of the cycle, the knee joint flexion angle increased, the femorotibial contact forces moved in posterior direction (Freeman 2001) and decreased (Taylor et al. 2004) leading to less constraining loading of the bone, wedge and plate. The surgical intervention induced-loading was approximate because of the simplified soft tissue description. It was not considered that during the opening process of the osteotomy, the periosteum may be damaged, resulting in overestimated elongation loads. However, neglecting the variation of thickness and stiffness of the periosteum due to muscles and ligament attachment area underestimate the elongation loads. The elongation of the femorotibial soft tissues was not taken into account. Possible overestimation of the preload may be viewed as an additional safety margin in what concerns stress between the wedge 
and bone. However, the effect of the plate position may not be affected by these assumptions. Finally, the micromotions at the bone wedge interface were considered only after preload, thus minimizing the effect of preload on the micromotion magnitude.

Referring to the literature, three clinical studies (Hernigou and Ma 2001, Koshino et al. 2003, Staubli et al. 2003) mentioned an anteromedial plate position for medial opening wedge tibial osteotomy. However, these studies like the numerical ones (Sato and Morishita 1999, Schmitt et al. 2001) found in the literature did not identify the biomechanical advantages related to such a position. Furthermore, the impact of different plate positions in open wedge osteotomy was not assessed.

\section{Conclusions}

The present FEM study showed that the SP position had a great impact on the biomechanical behavior of the open wedge varus osteotomy under maximal gait load. Positioning the SP medially was found to support smaller loading than an open wedge osteotomy with anteromedial plate position, thus achieving smaller structural stability and safety. Furthermore, micromotions at the bone/wedge interface were found to be of greater magnitude when the plate was positioned medially rather than anteromedially. Finally, an open wedge osteotomy with an anteromedial plate should be loaded under $18.8 \%(\sim 50 \mathrm{~kg})$ of the normal gait load until the osteotomy interfaces union is achieved. It should avoid fibrous tissue formation at the bone/wedge interface.

\section{Acknowledgements}

This research was supported by the grants from the Swiss National Science Foundation (FNRS No. 2100066872.01/1), the Fondation Lémanique pour la Recherche sur le Tissu Osseux and the Lausanne Center for Bone Tissue Engineering.

\section{References}

N.P. Badhe and I.W. Forster, "High tibial osteotomy in knee instability: the rationale of treatment and early results", Knee Surgery Sports Traumatol. Arthroscopy, 10(1), pp. 38-43, 2002.

P. Büchler, et al. "A finite element model of the shoulder: Application to the comparison of normal and osteoarthritic joints", Clin. Biomech., 17(9-10), pp. 630-639, 2002.

S.C. Cowin, Bone Mechanics Handbook, 2nd ed., Boca Raton, CRC Press, 2001.

T.L.H. Donahue, et al. "How the stiffness of meniscal attachments and meniscal material properties affect tibio-femoral pressure computed using a validated finite element model of the human knee joint", J. Biomech., 36(1), pp. 19-34, 2003.

M.A.R. Freeman, "How the knee moves", Curr. Orthopaedics, 15(6), pp. 444-450, 2001.

L. Han, J.A. Noble and M. Burcher, "A novel ultrasound indentation system for measuring biomechanical properties of in vivo soft tissue", Ultrasound Med. Biol., 29(6), pp. 813-823, 2003.
E.J. Harper and W. Bonfield, "Tensile characteristics of ten commercial acrylic bone cements", J. Biomed. Materials Research, 53(5), pp. 605-616, 2000.

P. Hernigou and W. Ma, "Open wedge tibial osteotomy with acrylic bone cement as bone substitute", The Knee, 8(2), pp. 103-110, 2001.

P. Hernigou, et al. "Proximal tibial osteotomy for osteoarthritis with varus deformity. A ten to thirteen-year follow-up study", J. Bone Joint Surg., Am. vol., 69(3), pp. 332-354, 1987.

P.F. Hill, et al. "Tibiofemoral movement 2: the loaded and unloaded living knee studied by MRI", J. Bone Joint Surg., Br. vol., 82(8), pp. 1196-1198, 2000.

D.E. Hurwitz, et al. "Dynamic knee loads during gait predict proximal tibial bone distribution", J. Biomech., 31(5), pp. 423-430, 1998.

M. Jasty, et al. "In vivo skeletal responses to porous-surfaced implants subjected to small induced motions", J. Bone Joint Surg., Am. vol., 79(5), pp. 707-714, 1997.

T. Koshino, T. Murase and T. Saito, "Medial opening-wedge high tibial osteotomy with use of porous hydroxyapatite to treat medial compartment osteoarthritis of the knee", J. Bone Joint Surg., Am. vol., 85-A(1), pp. 78-85, 2003.

F. Linde, et al. "Mechanical properties of trabecular bone. Dependency on strain rate", J. Biomech., 24(9), pp. 803-809, 1991.

P. Lobenhoffer, J. Agneskirchner and W. Zoch, "Open-wedge high tibial osteotomy with special medial plate fixator", Der Orthopaede, 33(2), pp. 153-160, 2004.

R.M. Pilliar, J.M. Lee and C. Maniatopoulos, "Observations on the effect of movement on bone ingrowth into porous-surfaced implants", Clin. Orthopaedics Related Res., (208), pp. 108-113, 1986.

D.P. Pioletti, et al. "Intrinsic coordinate system for the tibial plateau", The Knee, 5(2), pp. 95-98, 1998a.

D.P. Pioletti, et al. "Viscoelastic constitutive law in large deformations: application to human knee ligaments and tendons", J. Biomech., 31(8), pp. 753-757, 1998b.

T.E. Popowics, Z. Zhu and S.W. Herring, "Mechanical properties of the periosteum in the pig, Sus scrofa", Arch. Oral Bio., 47(10), pp. 733-741, 2002.

R.L. Rakotomanana, et al. "A finite-element model for evaluation of tibial prosthesis bone interface in total knee replacement", J. Biomech., 25(12), pp. 1413-1424, 1992.

N.A. Ramaniraka, et al. A Numerical Study on the Influence of Graft Mechanical Properties after Posterior Cruciate (PCL) Reconstruction in International Congress on Computational Bioengineering, 2003, pp. 417-422.

D.T. Reilly and A.H. Burstein, "The elastic and ultimate properties of compact bone tissue", J. Biomech., 8(6), pp. 393-405, 1975.

J.C. Rice, S.C. Cowin and J.A. Bowman, "On the dependence of the elasticity and strength of cancellous bone on apparent density", J. Biomech., 21(2), pp. 155-168, 1988.

P.J. Rubin, et al. "Frictional interface micromotions and anisotropic stress-distribution in a femoral total hip component", J. Biomech., 26(6), pp. 725-739, 1993.

H. Sato and S. Morishita, "Effect of quadriceps exercise on synostosis following tibial osteotomy with internal fixation: a finite element simulation", Clin. Biomech., 14(1), pp. 1-6, 1999.

J. Schmitt, J. Meiforth and M. Lengsfeld, "Development of a hybrid finite element model for individual simulation of intertrochanteric osteotomies", Med. Eng. Phys., 23(8), pp. 529-539, 2001.

J.S. Shockey, J.A.v. Fraunhofer and D. Seligson, "A measurement of the coefficient of static friction of human long bones", Surf. Technol., 25(2), pp. 167-173, 1985.

K. Soballe, "Hydroxyapatite ceramic coating for bone implant fixation. Mechanical and histological studies in dogs", Act. Orthopaedica Scandinavica, 255, pp. 1-58, 1993.

K. Soballe, et al. "Tissue ingrowth into titanium and hydroxyapatitecoated implants during stable and unstable mechanical conditions", J. Orthopaedic Res., 10(2), pp. 285-299, 1992a.

$\mathrm{K}$. Soballe, et al. "Hydroxyapatite coating modifies implant membrane formation. Controlled micromotion studied in dogs", Act. Orthopaedica Scandinavica, 63(2), pp. 128-140, 1992b.

A.E. Staubli, et al. "TomoFix: a new LCP-concept for open wedge osteotomy of the medial proximal tibia-early results in 92 cases", Inj., 34(Suppl 2), pp. B55-B62, 2003.

S.J. Taylor, et al. "Telemetry of forces from proximal femoral replacements and relevance to fixation", J. Biomech., 30(3), pp. 225-234, 1997.

W.R. Taylor, et al. "Tibio-femoral loading during human gait and stair climbing", J. Orthopaedic Res., 22(3), pp. 625-632, 2004. 
E. Uchiyama, K. Yamakoshi and T. Sasaki, "Measurement of mechanical characteristics of tibial periosteum and evaluation of local differences", J. Biomech. Eng., 120(1), pp. 85-91, 1998.

J.A. Von Fraunhofer D.S. and L.A. Schaper, "The rotational friction characteristics of human long bones", Surf. Technol., 25(4), pp. $377-388,1985$.
J.L. Williams and J.L. Lewis, "Properties and an anisotropic model of cancellous bone from the proximal tibial epiphysis", J. Biomech. Eng., 104(1), pp. 50-56, 1982.

P. Wretenberg, D.K. Ramsey and G. Nemeth, "Tibiofemoral contact points relative to flexion angle measured with MRI", Clin. Biomech., 17(6), pp. 477-485, 2002. 